\title{
PAPER Selecting Effective and Discriminative Spatio-Temporal Interest Points for Recognizing Human Action
}

\author{
Hongbo ZHANG ${ }^{\dagger}$, Shaozi $\mathrm{LI}^{\dagger}$, Songzhi $\mathrm{SU}^{\dagger}$, Nonmembers, and Shu-Yuan $\mathrm{CHEN}^{\dagger \dagger \mathrm{a})}$, Member
}

\begin{abstract}
SUMMARY Many successful methods for recognizing human action are spatio-temporal interest point (STIP) based methods. Given a test video sequence, for a matching-based method using a voting mechanism, each test STIP casts a vote for each action class based on its mutual information with respect to the respective class, which is measured in terms of class likelihood probability. Therefore, two issues should be addressed to improve the accuracy of action recognition. First, effective STIPs in the training set must be selected as references for accurately estimating probability. Second, discriminative STIPs in the test set must be selected for voting. This work uses $\varepsilon$-nearest neighbors as effective STIPs for estimating the class probability and uses a variance filter for selecting discriminative STIPs. Experimental results verify that the proposed method is more accurate than existing action recognition methods.

key words: human action recognition, discriminative power, $\varepsilon-N N$ probability estimation, class likelihood probability, variance filter
\end{abstract}

\section{Introduction}

The recognition of action in video sequences is an active and challenging area of research in the field of computer vision [1], [2]. Human action recognition is the preliminary task in many applications, including video retrieval, intelligent vehicles, video surveillance and video event analysis. Methods for recognizing human action fall into two categories: top-down and bottom-up.

Top-down methods detect objects, categorize them and then describe their motion. Finally, based on the characteristics of motion, a model of action is established. Bobick and Davis [3] used the motion energy image (MEI) and the motion history image (MHI) to describe human motion. Recently, Wang et al. [4], Weiland et al. [5], Wang et al. [6] and Hsieh et al. [7] developed top-down methods based on the detection of human silhouettes or contours. These methods are partially effective because accurate recognition depends on accurate object detection which is yet an ill-posed problem.

Inspired by object recognition in image processing, bottom-up methods treat human action recognition as object recognition in the spatio-temporal dimension. Local features of interest points are first detected and represented.

Manuscript received August 23, 2012.

Manuscript revised December 21, 2012.

†The authors are with the School of Information Science and Technology, Xiamen University, Xiamen, China and Fujian Key Laboratory of the Brain-like Intelligent Systems, Xiamen University, China.

${ }^{\dagger \dagger}$ The author is with the Department of Computer Science and Engineering, Yuan Ze University, Taiwan.

a) E-mail: cschen@ saturn.yzu.edu.tw (Corresponding author) DOI: 10.1587/transinf.E96.D.1783
An action is then defined as a set of features. Finally, an "action object" is established as a recognition model. Based on this idea, many researchers used object recognition methods in image processing to recognize human action yielding some new "human action object" recognition methods. Interest points for recognizing actions are widely detected by a 3D Harris spatio-temporal interest point (STIP) detector, which was extended from Harris corner detection [9] to three-dimensional space by Laptev et al. [8]. Other STIP detectors include the cuboid detector [10], the feature detector based on the Hessian matrix [11] and the three-dimensional SIFT detector [12].

Niebles et al. [13] and Liu et al. [14] quantified STIPs as spatio-temporal visual words to generate STIP vocabularies and used the bags-of-words (BOW) model for recognizing actions. Wang et al. [15] extracted video blocks at regular positions and scales in spatio-temporal space by dense sampling and then used the BOW model for recognizing actions. Methods based on the gradient or optical flow are extensively used for representing STIPs. Laptev et al. [8] used the N-jets methods [16], based on image gradient and optical flow, to describe STIP as a histogram of oriented gradient (HOG) and an histogram of optical flow (HOF) [17]. Other descriptors include the 3D-SIFT descriptor [12], the 3D-HOG descriptor [18] and the ESURF descriptor [19].

Most action classification algorithms that are based on STIP description apply learning-based algorithms. These include SVM [20], Boosting [19], the generative model that is based on some parameter estimations [21] and others. These algorithms have training and learning phases. Whenever the training set is changed by an increase or decrease in the number of samples or the addition of new categories, retraining is required. To prevent retraining, Yuan et al. [23], inspired by the NBNN algorithm [22] for image classification, proposed the Native Bayes Mutual Information Maximization (NBMIM) algorithm for classifying actions. The NBMIM method identifies the class of a given test sequence as the one having the maximum decision score, which is obtained by summing scores casted from all the STIPs in the action sequence. The decision score for each STIP is based on point-wise mutual information between the STIP and all of STIPs in the training set. Yuan et al. [23] achieved an accuracy of $93.7 \%$ using 16 training subjects, which exceeded the accuracies achieved using other methods when applied to the same dataset [10], [15], [21], [24], [25].

In the NBMIM algorithm, the distance between a STIP and a class is measured in terms of mutual information. 
Therefore, recognition accuracy depends on precise estimation of the class conditional probability. NBMIM used 1-NN (Nearest neighbor) for probability estimation; however, to achieve an efficient 1-NN search, Yuan et al. [23] used $\varepsilon$-NN search to prevent global nearest neighbor searching. Since precise non-parameter estimation of class probability is based on the fact that numerous training samples are available, an estimation algorithm, which can work well even with few training samples, should be proposed. To this end, this work uses the $\varepsilon$-NN probability estimation method.

Various actions such as running and jogging actions may have similar local STIPs. In other words, the same STIPs may exist in different action categories yielding nonempty intersection of STIP sets between different classes. STIPs that have similar distances to different action categories are regarded as indiscriminative STIPs and should be removed to improve the accuracy of action recognition. To do so, the work proposes the variance filter for STIP selection. In summary, this work proposed the $\varepsilon$-NN probability estimation method and the variance filter for discriminative STIP selection. Various experiments are performed on the KTH database to verify that NBMIM with the $\varepsilon$-NN probability estimation method and the variance filter for STIP selection outperforms the existing methods based on STIPs.

In the following, Sect. 2 introduces action classification problem and the KTH database on which this work is based. Section 3 describes the algorithms on which the proposed method is based. Section 4 presents and discusses relevant experimental results. Section 5 concludes the work and proposes future work.

\section{Action Classification}

\subsection{Problem Definition}

The problem of action recognition is to identify the action that is performed in a given video sequence. Among various action recognition methods, the STIP method has achieved considerable success in recent years. In this method, a video is represented by a set of STIPs. The action recognition problem is then formulated as follows: Given a video sequence $Q$, a set of $C$ action categories $\{1,2, \cdots, C\}$, and a training set of labeled videos $T=\bigcup_{c=1, \cdots, C ; n=1, \cdots, N} T_{n}^{c}$, the category of video is identified. Here, $N$ is the number of labeled videos for each category, so the training set $T$ has $C \times N$ video sequences. In the STIP-based method, the video sequence $Q$ is represented by a collection of STIPs that are detected from each frame of the video, and denoted by $Q=\left\{d_{q}\right\}$. Similarly, the training set $T$ is denoted by $T=\left\{d_{t}^{c}\right\}=\bigcup_{c=1, \cdots, C} T^{c}$ with $T^{c}=\left\{d_{t}^{c} \mid d_{t}^{c} \in \underset{n=1, \cdots, N}{\bigcup} T_{n}^{c}\right\}$.

\subsection{Database}

The proposed method is designed based on the KTH dataset [26], which contains six actions $(C=6)$ : "walking", "jogging", "running", "boxing", "handwaving", and

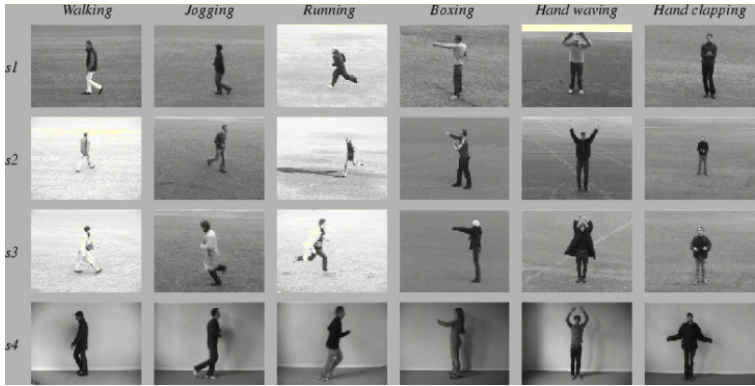

Fig. 1 Examples of KTH dataset [26].

"handclapping". Each action has 25 subjects in 4 different environments $(E=4)$ - outdoors, outdoors with variable scales, outdoors with different clothes, and indoors with variable lighting conditions. In other words, there are $C \times E=6 \times 4=24$ action videos related to a subject. Figure 1 presents some examples of actions. In this work, STIPs are detected by the 3D-Harris detector and represented by concatenating HOG and HOF features (162-dimensional feature vector), as in NBMIM [23]. However, to verify our method in practical use, another two datasets were used to demonstrate the performance of our method as described in Sect. 4.

\section{Classification Method}

\subsection{NBMIM Algorithm}

The NBMIM algorithm [23] encodes $Q=\left\{d_{q}\right\}$ as a collection of 3D-Harris interest points [8], which are represented by two descriptors: HOG and HOF [17]. In other words, $d_{q}$ is the feature vector that represents an STIP. The NBMIM algorithm treats action recognition as an optimization problem. The class of test action $Q$ is the class $c^{*}$ that has the maximum voting score based on the mutual information between $Q$ and a specific class $c$ corresponding to the following equation.

$$
\begin{aligned}
c^{*}=\arg \max _{c \in\{1,2, \cdots, C\}} M I(\hat{c}=c, Q)=\arg \max _{c \in\{1,2, \cdots, C\}} \sum_{d_{q} \in Q} s^{c}\left(d_{q}\right) \\
s^{c}\left(d_{q}\right)=\operatorname{MI}\left(\hat{c}=c, d_{q}\right)=\log \frac{p\left(d_{q} \mid \hat{c}=c\right)}{p\left(d_{q}\right)} \\
=\log \frac{p\left(d_{q} \mid \hat{c}=c\right)}{p\left(d_{q} \mid \hat{c}=c\right) P(\hat{c}=c)+p\left(d_{q} \mid \hat{c} \neq c\right) P(\hat{c} \neq c)} \\
=\log \frac{C}{1+\frac{p\left(d_{q} \mid \hat{c} \neq c\right)}{p\left(d_{q} \mid \hat{c}=c\right)}(C-1)}
\end{aligned}
$$

where $s^{c}\left(d_{q}\right)=\operatorname{MI}\left(\hat{c}=c, d_{q}\right)$ is the mutual information score between $d_{q}$ and the specific action category $c ; c \in$ $\{1,2, \cdots, C\}$ is a class label, and $P(\hat{c}=c)=\frac{1}{C}$ is the equal prior probability. Notably, the equation $s^{c}\left(d_{q}\right)=$ $M I\left(\hat{c}=c, d_{q}\right)>0$ implies that the likelihood ratio in terms of conditional class probabilities, $\frac{p\left(d_{q} \mid \hat{c} \neq c\right)}{p\left(d_{q} \mid \hat{c}=c\right)}$ is less 
than one and $d_{q}$ votes positively; in contrast, the equation $s^{c}\left(d_{q}\right)=M I\left(\hat{c}=c, d_{q}\right)<0$ implies that the likelihood ratio $\frac{p\left(d_{q} \mid \hat{c} \neq c\right)}{p\left(d_{q} \mid \hat{c}=c\right)}$ is greater than one and $d_{q}$ votes negatively.

Let all of the negative STIP samples for class $c$ be denoted by $T^{c-}, T^{c-}=\bigcup_{\hat{c} \in\{1,2, \cdots, C\} \wedge \hat{c} \neq c} T^{\hat{c}}$, and all of the positive STIP samples for class $c$ be denoted as $T^{c}$, as defined in Sect. 2.1. In computing the conditional class probability $p\left(d_{q} \mid \hat{c}=c\right)$, samples $d_{t}^{c}$ in the positive dataset $\left(T^{c}\right)$ with distances to $d_{q}$ less $\varepsilon$ are first collected to obtain $N N_{\varepsilon}^{c}\left(d_{q}\right)$. Similarly, samples in the negative dataset $T^{c-}$ are also collected to obtain $N N_{\varepsilon}^{c-}\left(d_{q}\right)$. NBMIM then uses the nearest neighbor approximation to estimate the likelihood ratio by using the following equation:

$$
\begin{aligned}
& \frac{p\left(d_{q} \mid \hat{c} \neq c\right)}{p\left(d_{q} \mid \hat{c}=c\right)}=\frac{\left|T^{c}\right| \sum_{d_{t}^{\hat{c} \in T T^{c}}} K\left(d_{q}-d_{t}^{\hat{c}}\right)}{\left|T^{c-}\right| \sum_{d_{t}^{c} \in T^{c}} K\left(d_{q}-d_{t}^{c}\right)} \\
& \approx \frac{\exp \left[-\frac{1}{2 \sigma^{2}}\left(\left\|d_{q}-d_{N N}^{c-}\left(d_{q}\right)\right\|^{2}\right)\right]}{\exp \left[-\frac{1}{2 \sigma^{2}}\left(\left\|d_{q}-d_{N N}^{c}\left(d_{q}\right)\right\|^{2}\right)\right]} \\
& =\exp \left[-\frac{1}{2 \sigma^{2}}\left(\left\|d_{q}-d_{N N}^{c-}\left(d_{q}\right)\right\|^{2}-\left\|d_{q}-d_{N N}^{c}\left(d_{q}\right)\right\|^{2}\right)\right] \\
& \left\|d_{q}-d_{N N}^{c-}\left(d_{q}\right)\right\|=\min _{d_{t}^{c} \in N N_{\varepsilon}^{c-}\left(d_{q}\right)}\left\|d_{q}-d_{t}^{\hat{c}}\right\|, \\
& \left\|d_{q}-d_{N N}^{c}\left(d_{q}\right)\right\|=\min _{d_{t}^{c} \in N N_{\varepsilon}^{c}\left(d_{q}\right)}\left\|d_{q}-d_{t}^{c}\right\|
\end{aligned}
$$

where $d_{N N}^{c-}\left(d_{q}\right)$ and $d_{N N}^{c}\left(d_{q}\right)$ are the nearest neighbors of $d_{q}$ in the negative and positive datasets, respectively. The best bandwidth $\sigma$ is selected by adjusting the value $\sigma$ based on the purity in the neighborhood of the STIP $d_{q}$ in NBMIM using the following equation:

$$
\begin{gathered}
\frac{1}{2 \sigma^{2}}=\left\{\begin{array}{cc}
\frac{\left|N N_{\varepsilon}^{c}\left(d_{q}\right)\right|}{\left|N N_{\varepsilon}^{c}\left(d_{q}\right)\right|+\left|N N_{\varepsilon}^{c-}\left(d_{q}\right)\right|} & \gamma(d) \geq 0 \\
\frac{\left|N N_{\varepsilon}^{c-}(d)\right|}{\left|N N_{\varepsilon}^{c}\left(d_{q}\right)\right|+\left|N N_{\varepsilon}^{c-}\left(d_{q}\right)\right|} & \gamma(d) \leq 0
\end{array}\right. \\
\gamma(d)=\left\|d_{q}-d_{N N}^{c-}\left(d_{q}\right)\right\|^{2}-\left\|d_{q}-d_{N N}^{c}\left(d_{q}\right)\right\|^{2}
\end{gathered}
$$

\section{$3.2 \varepsilon$-NN Probability Estimation Method}

The proposed method uses $\varepsilon$-NN rather than 1-NN as used in NBMIM to estimate the likelihood ratio according to the following equation:

$$
\begin{aligned}
& \frac{p\left(d_{q} \mid \hat{c} \neq c\right)}{p\left(d_{q} \mid \hat{c}=c\right)}=\frac{\left|T^{c}\right| \sum_{d_{t}^{\hat{c}} \in T^{c-}} K\left(d_{q}-d_{t}^{\hat{c}}\right)}{\left|T^{c-}\right| \sum_{d_{t}^{c} \in T^{c}} K\left(d_{q}-d_{t}^{c}\right)} \\
& \approx \frac{\left|N N_{\varepsilon}^{c}\left(d_{q}\right)\right| \sum_{d_{t}^{\hat{c}} \in N N_{\varepsilon}^{c-}\left(d_{q}\right)} K\left(d_{q}-d_{t}^{\hat{c}}\right)}{\left|N N_{\varepsilon}^{c-}\left(d_{q}\right)\right| \sum_{d_{t}^{c} \in N N_{\varepsilon}^{c}\left(d_{q}\right)} K\left(d_{q}-d_{t}^{c}\right)}
\end{aligned}
$$

$$
\approx \frac{\left|N N_{\varepsilon}^{c}\left(d_{q}\right)\right| \sum_{d_{t}^{\hat{c}} \in N N_{\varepsilon}^{c-}\left(d_{q}\right)} \exp \left[-\frac{1}{2 \sigma^{2}}\left(\left\|d_{q}-d_{t}^{\hat{c}}\right\|^{2}\right)\right]}{\left|N N_{\varepsilon}^{c-}\left(d_{q}\right)\right| \sum_{d_{t}^{c} \in N N_{\varepsilon}^{c}\left(d_{q}\right)} \exp \left[-\frac{1}{2 \sigma^{2}}\left(\left\|d_{q}-d_{t}^{c}\right\|^{2}\right)\right]}
$$

Additionally, the proposed method seeks a fixed bandwidth $\sigma$ empirically rather than by adaptive adjustment of $\sigma$ in NBMIM to eliminate the need for adaptive computation. In this work, $\sigma$ is empirically set to 0.4 , which yields the best accuracy, as demonstrated by the experimental results.

The reason for the use of $\varepsilon$-NNs rather than 1-NN to estimate the conditional class probability for likelihood ratio is as follows. A higher error rate is obtained when probability estimation is based on 1-NN, particularly when the number of training samples is small. The experimental results as described in Sect. 4 support this claim. Therefore, $\varepsilon$-NN rather than 1-NN is used for probability estimation in this work.

NBMIM used 1-NN for probability estimation, however, to achieve an efficient 1-NN search, Yuan et al. [23] used $\varepsilon$-NN search to prevent global nearest neighbor searching. The experimental results of Yuan et al. [23] reveal that setting $\varepsilon$ to 2.2 yields the highest accuracy; nevertheless, classification performance is insensitive to the choice of $\varepsilon$. The same conclusion is drawn in this work, and so $\varepsilon$ is also set to 2.2 .

\subsection{Variance Filter for STIP Selection}

The decision function of action sequence $Q=\left\{d_{q}\right\}$ is the sum of voting scores $s^{c}\left(d_{q}\right)$ in terms of mutual information between all STIPs, $d_{q}$ and the action category $c$. However, selecting discriminative STIPs, $d_{q}$, for voting is critical to improving classification accuracy. In this work, the discriminative power of each STIP is measured as the difference between the mutual information of the STIP and all of the action categories. Clearly, a smaller difference between the mutual information of the STIP and various categories is associated with less discriminative power. In other words, the variance of $s^{c}\left(d_{q}\right)=M I\left(\hat{c}=c, d_{q}\right)$, denoted by $\operatorname{Var}\left(d_{q}\right)$, is used to measure the discriminative power of $d_{q}$ and is given by the following equation:

$$
\operatorname{Var}\left(d_{q}\right)=\frac{1}{C} \sum_{\hat{c}=1}^{C}\left(s^{\hat{c}}\left(d_{q}\right)-\frac{1}{C} \sum_{j=1}^{C} s^{j}\left(d_{q}\right)\right)^{2}
$$

The final decision function of the proposed method is modified from Eq. (1), used in NBMIM [23], to form the following equation:

$$
\begin{gathered}
c^{*}=\arg \max _{c \in\{1,2, \cdots, C\}} \sum_{d_{q} \in Q} w\left(d_{q}\right) s^{c}\left(d_{q}\right), \\
w\left(d_{q}\right)= \begin{cases}1 & \operatorname{Var}\left(d_{q}\right)>\operatorname{Var}_{h} \\
0 & \operatorname{Var}\left(d_{q}\right) \leq \operatorname{Var}_{h}\end{cases}
\end{gathered}
$$

where $\operatorname{Var}_{h}$ is a threshold value. The threshold value of $\operatorname{Var}_{h}$ is critical to maximizing accuracy and is determined empirically on the KTH database as described in Sect. 3.4. 
In summary, there are eight versions of NBMIM according to the bandwidth, the methods for probability estimation, and whether or not the variance filter is used. The methods to which are attached "_f” or "_a" use fixed or adaptive bandwidth, respectively, the methods to which are attached "+1-NN" or " $+\varepsilon$-NN" use 1-NN or $\varepsilon$-NN for probability estimation, respectively, and the methods to which are attached "+Var" use the variance filter to select discriminative STIPs for voting. Therefore, NBMIM_a+1-NN denotes the original version of NBMIM, and NBMIM $\_+\varepsilon-\mathrm{NN}+\mathrm{Var}$ denotes the proposed version of NBMIM.

\subsection{Threshold Selection for Variance Filter}

The performance of action recognition is defined in terms of precision $(P)$, recall $(R)$, and F-score. The $P$ and $R$ values are determined from the recall and precision variables for each action $c, P_{c}$ and $R_{c}$, using the equations $P_{c}=\frac{t p}{t p+f p}$, $R_{c}=\frac{t p}{t p+f n}$, where $t p$ denotes the number of videos whose recognized action and ground truth are both $c ; f p$ is the number of videos whose recognized action is $c$ but whose ground truth is not $c ; f n$ is the number of videos whose recognized action is not $c$ but whose ground truth is $c$. The values of $P$ and $R$ can then be defined as the mean values of $P_{c}$ and $R_{c}$ over the $C$ actions, in this work $C$ is set to 6 . Notably, recall is equal to recognition accuracy used for performance evaluation of human action recognition [23].

Let the number of selected subjects for training be $S$, $S$ subjects are randomly selected and their corresponding actions are collected as a training dataset; the remaining videos are used in a test. If eight persons are selected for training $(S=8)$, then the number of labeled videos in each category, $N$, is set to $S \times E=8 \times 4=32$ and the training dataset contains $C \times S \times E=6 \times 32=192$ videos. The remaining $6 \times 17 \times 4=408$ action videos, corresponding to the other 17 subjects, are regarded as the test dataset.

The threshold value of $\mathrm{Var}_{h}$ for the variance filter is determined by performing $S$-fold validation on the training dataset $T=\left\{d_{t}^{c}\right\}$ with $S$ subjects. The details are described as follows:

For a run in the $S$-fold validation on the training dataset $T=\left\{d_{t}^{c}\right\}$, assume that subject $s$ is selected for validation. The validation set, denoted by $T_{s}=\bigcup_{c=1, \ldots, C, e=1, \ldots, E}\left\{d_{v}\right\}_{s}^{c, e}$, includes all of the action sequences that are related to subject $s$. Here, $C=6$ and $E=4$ for the KTH dataset. In other words, $T_{s}$ comprises 24 action sequences. For each STIP, $d_{v}$ of the action sequences $\left\{d_{v}\right\}_{s}^{c, e}$ in the set $T_{s}$, the voting scores $S^{c}\left(d_{v}\right)$ for the specific class $c$ are given by Eqs. (2) and (5) and the score variance $\operatorname{Var}\left(d_{v}\right)$ is then computed using Eq. (6). All of the values of $\operatorname{Var}\left(d_{v}\right)$ for the STIP, $d_{v}$, are then recorded as a range and denoted by $\operatorname{Var}_{h}\left(d_{v}\right) \in\left[\operatorname{Var}_{\min }\left(d_{v}\right), \operatorname{Var}_{\max }\left(d_{v}\right)\right]$. The union of $\left[\operatorname{Var}_{\min }\left(d_{v}\right), \operatorname{Var}_{\max }\left(d_{v}\right)\right]$ that corresponds to the respective STIPs, $d_{v}$, for the action sequences $\left\{d_{v}\right\}_{s}^{c, n}$ in the set $T_{s}$ are then recorded as $\left[\operatorname{Var}_{\min }^{s}, \operatorname{Var}_{\max }^{s}\right]$. Action recognition is performed for the action sequence $\left\{d_{v}\right\}_{s}^{c, e}$ in the set $T_{s}$ using $\mathrm{NBMIM}+\varepsilon-\mathrm{NN}+$ Var with training dataset that includes the other $S-1$ subjects and various values of $\operatorname{Var}_{h}$ set in the range $\left[V a r_{\min }^{s}, \operatorname{Var}_{\max }^{s}\right]$. The mean accuracies for the 24 video sequences $\left\{d_{v}\right\}_{s}^{c, e}$ can then be obtained for various values of $\mathrm{Var}_{h}$. A curve of mean accuracies for the specific subject $s$ as a function of $\mathrm{Var}_{h}$ over the range [ $\mathrm{Var}_{\min }^{s}, \mathrm{Var}_{\max }^{s}$ ] can then be plotted. For example, in the case of $S=24$ and $s=12$, its curve of mean accuracies is plotted as the blue curve in Fig. 2.

The $S$ mean curves of accuracies for the respective $S$ subjects are finally collected to yield the mean curve of accuracies for the entire training dataset. The final mean curve of accuracies for $S=24$ is plotted as the black curve and denoted by AVG in Fig. 2. The value of $V a r_{h}$ with the highest accuracy in the final mean curve of accuracies is then selected as the final threshold value of $\operatorname{Var}_{h}$. For example, the final threshold is set to 0.26 since the peak accuracy of 0.96 is reached at 0.26 in Fig. 2.

In this work, two other adaptive methods for selecting threshold are considered. First, the mean of variances is selected as the threshold. Second, the variances of STIPs

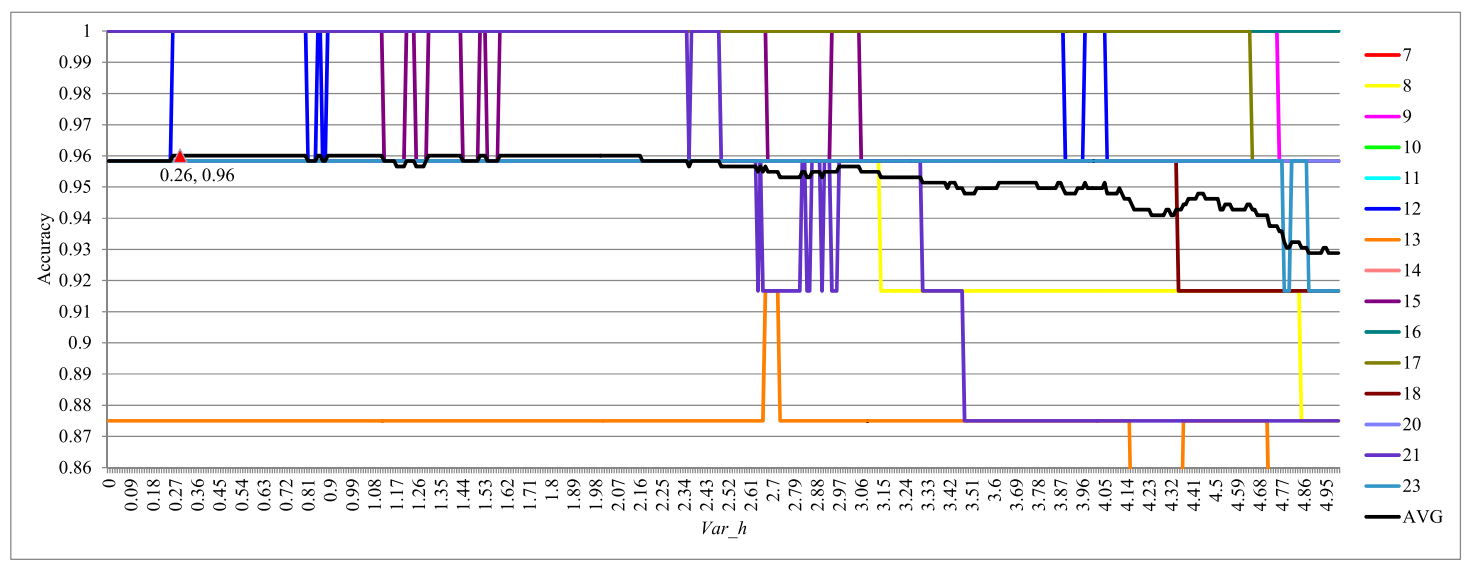

Fig. 2 Comparison of accuracies for various threshold values of $\operatorname{Var}_{h}$. 
Table 1 Comparison of accuracies achieved using various threshold selection methods.

\begin{tabular}{|c|c|c|c|c|c|c|c|}
\hline$S$ & 6 & 8 & 10 & 12 & 14 & 16 & 24 \\
\hline Optimal & $92.54 \%$ & $94.12 \%$ & $93.33 \%$ & $94.87 \%$ & $96.59 \%$ & $95.37 \%$ & $97.50 \%$ \\
\hline $\begin{array}{c}\text { Cross-validation } \\
\text { (NMBIM_f }+\varepsilon \text {-NN+Var) }\end{array}$ & $\mathbf{9 2 . 3 2} \%$ & $\mathbf{9 3 . 6 3} \%$ & $\mathbf{9 3 . 3 3 \%}$ & $\mathbf{9 4 . 8 7 \%}$ & $94.32 \%$ & $\mathbf{9 5 . 3 7 \%}$ & $\mathbf{9 7 . 0 0 \%}$ \\
\hline Mean & $91.67 \%$ & $92.65 \%$ & $91.67 \%$ & $92.63 \%$ & $\mathbf{9 4 . 7 0} \%$ & $92.59 \%$ & $96.17 \%$ \\
\hline Median & $92.32 \%$ & $93.14 \%$ & $92.50 \%$ & $94.87 \%$ & $94.32 \%$ & $94.91 \%$ & $96.17 \%$ \\
\hline NMBIM_f+ $\varepsilon$-NN & $92.32 \%$ & $93.38 \%$ & $93.06 \%$ & $94.55 \%$ & $94.32 \%$ & $93.98 \%$ & $96.50 \%$ \\
\hline
\end{tabular}

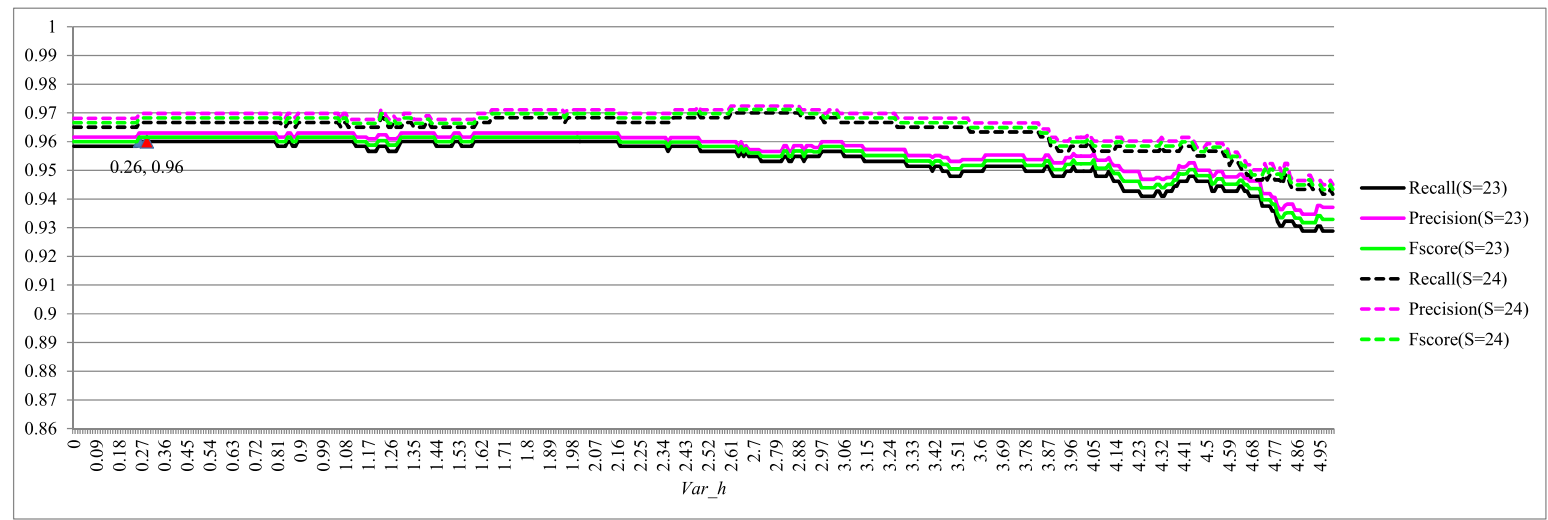

Fig. 3 Comparison of recall, precision, and F-score for various threshold values of $\operatorname{Var}_{h}$ with $S=23$ on training and $S=24$ on test sets, respectively.

are sorted in increasing order and the median variance is selected as the threshold. More specifically, the mean is defined individually for each test action sequence $Q=\left\{d_{q}\right\}$ in the test phase by $\operatorname{Var}_{h}=\sum_{d_{q} \in Q} \operatorname{Var}\left(d_{q}\right) /|Q|$, where $|Q|$ is the cardinality of $Q$. Similarly, the median is defined individually for each test action sequence $Q=\left\{d_{q}\right\}$ in the test phase by $\operatorname{Var}_{h}=\underset{d_{q} \in Q}{\operatorname{Median}}\left\{\operatorname{Var}\left(d_{q}\right)\right\}$.

Notably, the proposed cross-validation method selects a threshold value of $\mathrm{Var}_{h}$ for the entire action recognition system in the training phase. However, the mean and median strategies select threshold values of $\operatorname{Var}_{h}$ individually for each action sequence in the test phase. The latter strategies are more flexible than the former, but the former is more accurate (Table 1). Therefore, the former is used herein. The accuracies in Table 1 are computed by crossvalidation strategy. For example, in the case of $S=24$, 25 runs of action recognition are performed to calculate respective mean accuracies. Clearly, the accuracy is highest when the threshold value of $V r_{h}$ is determined by the cross-validation method in the training phase. The first row of Table 1 presents the optimal accuracies achieved using the NBMIM_f $+\varepsilon$-NN+Var algorithm for various numbers of training subjects. The optimal accuracies are obtained by performing exhaustive action recognition for various threshold values of $\operatorname{Var}_{h}$. Table 1 reveals that the accuracies achieved using cross-validation method are closest to the optimal accuracies.

In addition, Fig. 3 presents precision and recall rates achieved by NBMIM_f $+\varepsilon-\mathrm{NN}+$ Var for various threshold values of $\operatorname{Var}_{h}$ with $S=23$ on training and $S=23$ on test sets, respectively. Notably, the recall curve for the training set in Fig. 3 is the same as the AVG curve in Fig. 2. Figure 3 also shows that the proposed method is not sensitive to the threshold values of $\mathrm{Var}_{h}$ since the curves for test set $(S=24)$ is almost flat when the values of $\mathrm{Var}_{h}$ are set in the range [0.266, 3.87].

\section{Experiments}

Here, experiments were performed to verify the effectiveness of the proposed methods. Experimental results are obtained using the KTH dataset[26], the UCF sport dataset [30] and a new dataset collected from web site. Notably, parameters are determined based on the KTH dataset.

\subsection{Parameter Determination}

As mentioned in Sect. 3.2, the bandwidth $\sigma$ critically affects the accuracy of probability estimation. In this work, $\sigma$ is set to 0.4. This value is based on the obtained experimental results. In Table 2, the values of $\sigma$ range from 0.1 to 1 in steps of 0.2 , and this parameter varies with $S$, which is the number of subjects selected for training. Table 2 indicates that when $\sigma$ is set to 0.4 , the mean accuracies of NBMIM $f+\varepsilon-\mathrm{NN}$ are high for most values of $S$.

In addition, Fig. 4 shows the Receiver Operating Characteristic (ROC) curves achieved by NBMIM_f $+\varepsilon-\mathrm{NN}$ with $S=24$ for various bandwidth values of $\sigma$. To plot ROC curve for a specific bandwidth, a modified version of 
Table 2 Comparison of accuracies of NBMIM $\_+\varepsilon-\mathrm{NN}_{\mathrm{N}}$ for various bandwidth $\sigma$.

\begin{tabular}{|l|c|c|c|c|c|c|}
\hline$S$ & 1 & 0.8 & 0.6 & 0.4 & 0.2 & 0.1 \\
\hline 24 & $95.33 \%$ & $95.50 \%$ & $95.50 \%$ & $\mathbf{9 6 . 5 0} \%$ & $95.50 \%$ & $95.33 \%$ \\
\hline 16 & $93.98 \%$ & $93.98 \%$ & $\mathbf{9 4 . 4 4 \%}$ & $93.98 \%$ & $\mathbf{9 4 . 4 4 \%}$ & $\mathbf{9 4 . 4 4 \%}$ \\
\hline 14 & $92.80 \%$ & $92.80 \%$ & $\mathbf{9 4 . 7 0 \%}$ & $94.32 \%$ & $93.94 \%$ & $93.94 \%$ \\
\hline 12 & $92.31 \%$ & $92.31 \%$ & $93.27 \%$ & $\mathbf{9 4 . 5 5 \%}$ & $94.23 \%$ & $93.91 \%$ \\
\hline 10 & $92.22 \%$ & $92.22 \%$ & $\mathbf{9 3 . 0 6 \%}$ & $\mathbf{9 3 . 0 6} \%$ & $91.67 \%$ & $91.39 \%$ \\
\hline 8 & $91.42 \%$ & $91.67 \%$ & $92.40 \%$ & $\mathbf{9 3 . 3 8 \%}$ & $91.67 \%$ & $91.67 \%$ \\
\hline 6 & $90.57 \%$ & $90.35 \%$ & $91.01 \%$ & $\mathbf{9 2 . 3 2} \%$ & $90.57 \%$ & $89.91 \%$ \\
\hline
\end{tabular}

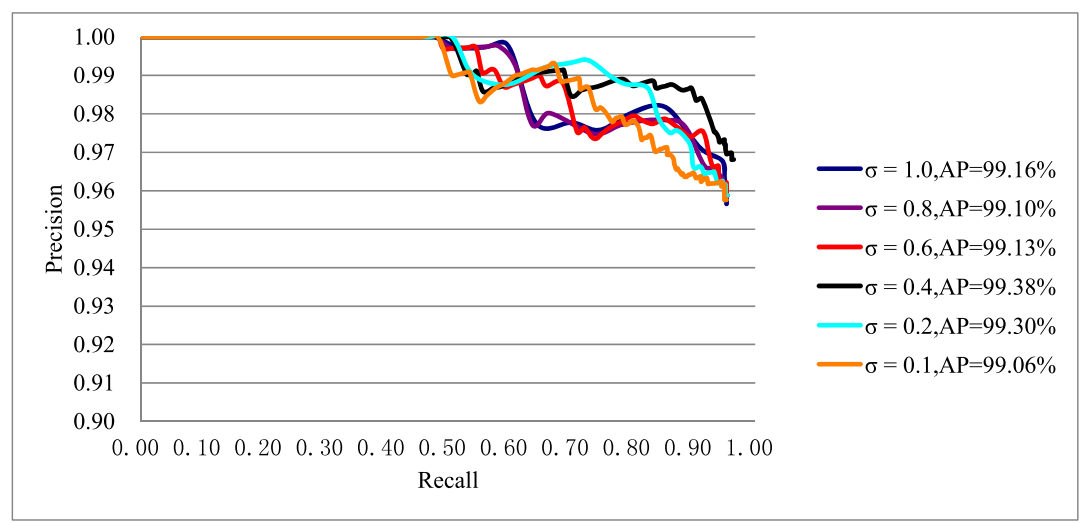

Fig. 4 ROC curves achieved by NBMIM_f $+\varepsilon$-NN with $S=24$ for various bandwidth $\sigma$.

Table 3 Comparison of accuracies achieved by NBMIM using 1-NN and $\varepsilon$-NN, with fixed and adaptive bandwidth $(\sigma)$, and with and w/o variance filter for various numbers of training subjects.

\begin{tabular}{|l|c|c|c|c|c|c|c|}
\hline \multicolumn{1}{|c|}{$S$} & 6 & 8 & 10 & 12 & 14 & 16 & 24 \\
\hline NMBIM_a+1-NN & $89.70 \%$ & $90.20 \%$ & $90.00 \%$ & $94.23 \%$ & $93.94 \%$ & $93.52 \%$ & $94.00 \%$ \\
\hline NMBIM_a+1-NN+Var & $91.45 \%$ & $91.42 \%$ & $91.67 \%$ & $94.70 \%$ & $\mathbf{9 4 . 3 2 \%}$ & $\mathbf{9 6 . 5 0 \%}$ & $96.00 \%$ \\
\hline NMBIM_f+1-NN & $90.35 \%$ & $91.18 \%$ & $91.11 \%$ & $93.92 \%$ & $93.56 \%$ & $93.98 \%$ & $96.17 \%$ \\
\hline NMBIM_f+1-NN+Var & $91.67 \%$ & $92.65 \%$ & $92.22 \%$ & $94.23 \%$ & $\mathbf{9 4 . 3 2 \%}$ & $94.44 \%$ & $96.17 \%$ \\
\hline NBMIM_a+ $\varepsilon$-NN & $89.70 \%$ & $91.18 \%$ & $90.83 \%$ & $93.27 \%$ & $92.80 \%$ & $92.59 \%$ & $96.33 \%$ \\
\hline NBMIM_a+ $\varepsilon$-NN+Var & $91.01 \%$ & $92.40 \%$ & $92.78 \%$ & $93.27 \%$ & $93.56 \%$ & $93.06 \%$ & $96.50 \%$ \\
\hline NBMIM_f+ $\varepsilon$-NN & $\mathbf{9 2 . 3 2} \%$ & $93.38 \%$ & $93.06 \%$ & $94.55 \%$ & $\mathbf{9 4 . 3 2 \%}$ & $93.98 \%$ & $96.50 \%$ \\
\hline NBMIM_f $+\varepsilon$-NN+Var & $\mathbf{9 2 . 3 2 \%}$ & $\mathbf{9 3 . 6 3 \%}$ & $\mathbf{9 3 . 3 3 \%}$ & $\mathbf{9 4 . 8 7 \%}$ & $\mathbf{9 4 . 3 2 \%}$ & $95.37 \%$ & $\mathbf{9 7 . 0 0 \%}$ \\
\hline
\end{tabular}

Eq. (7) with rejection is defined as follows:

$$
\begin{aligned}
& c^{*}= \\
& \begin{cases}\arg M A X & \text { if } M A X=\max _{c \in\{1,2, \cdots, C\}} \sum_{d_{q} \in Q} w\left(d_{q}\right) s^{c}\left(d_{q}\right)>\text { Score }_{h} \\
\text { reject } \quad \text { otherwise }\end{cases}
\end{aligned}
$$

The larger is the threshold value of Score $_{h}$, the lower is the recall rate. Therefore, the ROC curve is plotted in the increasing order of threshold values of Score $_{h}$. The average precision (AP), defined as the area under ROC curve, are $99.16,99.10,99.13,99.38,99.30$ and $99.06 \%$, for bandwidth $\sigma=1.0,0.8,0.6,0.4,0.2,0.1$, respectively. It is obvious that $\sigma=0.4$ has the highest AP value. Therefore, the fixed bandwidth $\sigma=0.4$ was used herein.

\subsection{Computational Complexity}

Notably, the computation complexities of various versions of NBMIM are almost balance. The reasons are as follows.
The intensive computation for various versions of NBMIM is to search nearest neighbors no matter $1-\mathrm{NN}$ or $\varepsilon-\mathrm{NN}$ since original NBMIM uses $\varepsilon$-NN search to achieve an efficient 1-NN search.

\subsection{Performance Evaluation of Action Recognition}

As described in Sect. 3.2 and 3.3, the $\varepsilon$-NN probability estimation method and variance filter for STIP selection are proposed to improve recognition accuracy. Here, some experiments are performed to prove the increase in accuracy, particularly for a small training set. Table 3 shows that NBMIM_f $+\varepsilon-N N+$ Var is typically the most accurate version of NBMIM, particularly in case of a small training set. The reasons are as follows. The increase from accuracy of the original NBMIM (NBMIM_a+1-NN) to that of the proposed method (NBMIM $\mathrm{f}+\varepsilon-\mathrm{NN}+\mathrm{Var}$ ) is over $3 \%$ in each case of the number of training samples less than ten; while the increases are only $1 \%$ in most cases that the numbers of training samples are greater than ten. 
Notably, NBMIM $f+1-N N$ (NBMIM $f+\varepsilon-N N)$ with fixed bandwidth outperforms NBMIM_a+1-NN (NBMIM_a $+\varepsilon$-NN) with adaptive bandwidth in most situations. Table 3 also demonstrates that even NBMIM_f $+\varepsilon-\mathrm{NN}$ is typically more accurate than the original version of NBMIM using 1-NN for probability estimation with fixed or adaptive bandwidth (NBMIM_a+1-NN, NBMIM_f+1-NN). Moreover, the versions with variance filter are better than those without variance filter in most situations. Therefore, the proposed method can select effective and discriminative STIPs to improve action recognition accuracy. Figure 5 presents the confusion matrix of NBMIM_f $+\varepsilon$-NN+Var for 24 training subjects. Finally, Table 4 compares the proposed method to the existing methods, which are boosted exemplar learning for action recognition and annotation [27], action recognition from one example [28], and action recognition by dense trajectories [29]. To the best of the authors' knowledge, the best accuracies of the-state-of-art methods for the KTH dataset with 24 training subjects are $95.33 \%$ [27], $95.10 \%$ [28], and $94.20 \%$ [29]. The method proposed herein has the highest accuracy of $97.00 \%$.

In order to verify our method in practical use, the proposed method was applied to the UCF sport dataset [30], which contains ten different types of sport actions: swinging (on the pommel horse and on the floor), diving, kicking (a ball), weight-lifting, horse-riding, running, skateboarding, high-swinging (at the high bar), golf swinging and walking. The dataset consists of 150 real videos with a large intra-class variability. In order to increase the amount of training samples, the dataset was added a horizontally flipped version of each video sequence to the dataset as used by Wang et al. [15]. Figure 6 presents some examples of actions.

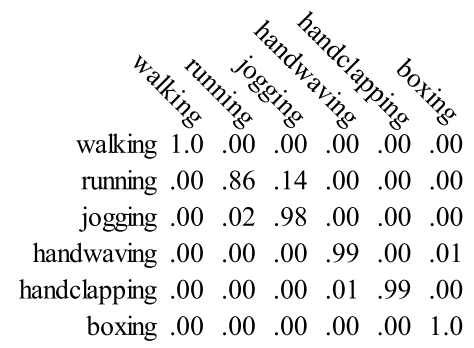

Fig. 5 Confusion matrix of NBMIM $\_+\varepsilon-N N+$ Var for 24 training subjects.
As similar to the experiments conducted by Wang et al. [15], the leave-one-out strategy is adopted to test on each original action sequence while the rest original video sequences together with their flipped versions are included as the training set. Note that the flipped version of the action sequence is removed from the test set as in [15]. Table 5 compares the proposed method to the existing methods, which are standard bag-of-feature SVM approach with

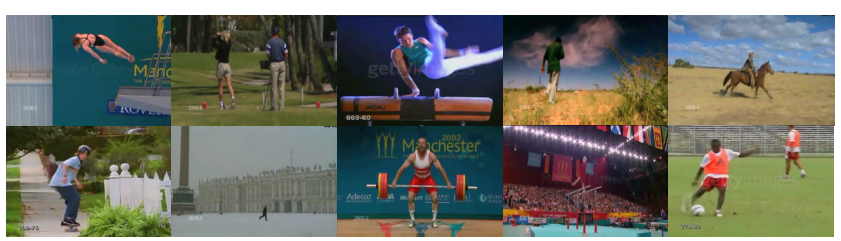

Fig. 6 Examples of UCF sports dataset. The actions from left to right and top to bottom are diving, golf swinging, swinging, walking, horse-riding, skateboarding, running, high-swinging, weight-lifting and kicking.

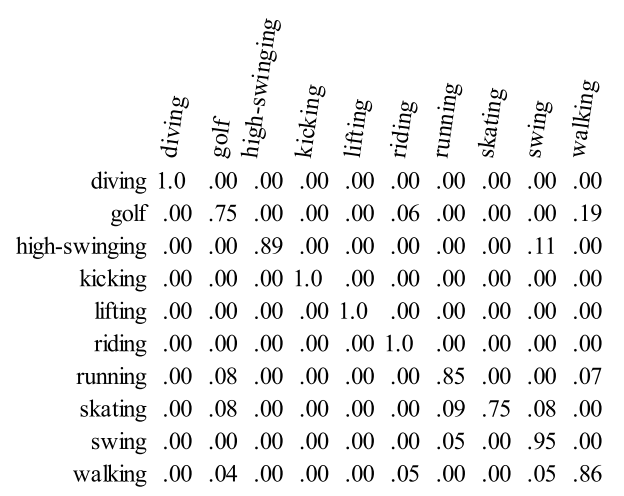

Fig. 7 Confusion matrix of NBMIM_f $+\varepsilon-\mathrm{NN}+$ Var for UCF sport dataset.

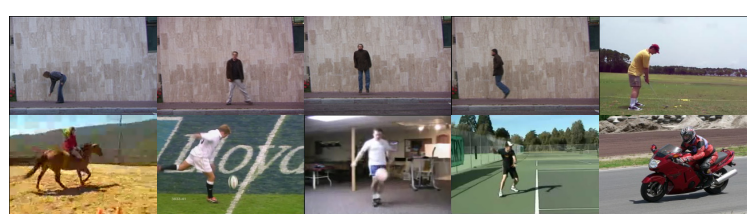

Fig. 8 Examples of a dataset containing other types of actions. The actions from left to right and top to bottom are (bend, jump, gallopingsideways, jump in place) from Weizmann dataset, (golf, riding horse, kicking) from UCF sport dataset, (juggle, tennis) from UCF YouTube dataset and riding motorbike through Google video retrieval.

Table 4 Comparison of method proposed herein with existing methods for 24 training subjects $(S=24)$.

\begin{tabular}{|l|c|c|c|c|c|c|}
\hline Methods & NBMIM_f+e -NN+Var & $\begin{array}{c}\text { NBMIM [23] } \\
\text { NBMIM_a+1-NN }\end{array}$ & $\begin{array}{c}\text { NBNN } \\
{[22]}\end{array}$ & $\begin{array}{c}\text { Zhang } \text { et } \\
\text { al. }[27]\end{array}$ & $\begin{array}{c}\text { Seo } \text { et al. } \\
{[28]}\end{array}$ & $\begin{array}{c}\text { Wang } \text { et } \\
\text { al. }[29]\end{array}$ \\
\hline Accuracy & $\mathbf{9 7 . 0 0 \%}$ & $94.00 \%$ & $92.83 \%$ & $95.33 \%$ & $95.10 \%$ & $94.20 \%$ \\
\hline
\end{tabular}

Table 5 Comparison of method proposed herein with existing methods for UCF sports dataset.

\begin{tabular}{|l|c|c|c|c|c|c|}
\hline Methods & NBMIM_f $+\varepsilon-N N+V a r$ & $\begin{array}{c}\text { NBMIM [23] } \\
\text { NBMIM_a+1-NN }\end{array}$ & $\begin{array}{c}\text { Wang } \text { et } \\
\text { al. }[15]\end{array}$ & $\begin{array}{c}\text { Rodriguez } \\
\text { et al. }[30]\end{array}$ & $\begin{array}{c}\text { Kovashka } \\
\text { et al. }[31]\end{array}$ & $\begin{array}{c}\text { Le } \text { et al. } \\
{[32]}\end{array}$ \\
\hline Accuracy & $\mathbf{9 0 . 4 9 \%}$ & $79.78 \%$ & $85.60 \%$ & $69.20 \%$ & $87.27 \%$ & $86.50 \%$ \\
\hline
\end{tabular}


dense 3D-HOG descriptors [15], maximum average correlation height filter for action recognition [30], learning a hierarchy of discriminative space-time neighborhood features for action recognition [31], and learning hierarchical invariant spatio-temporal features for action recognition with independent subspace analysis [32]. Our method has the highest accuracy of $90.49 \%$. Figure 7 presents the confu-

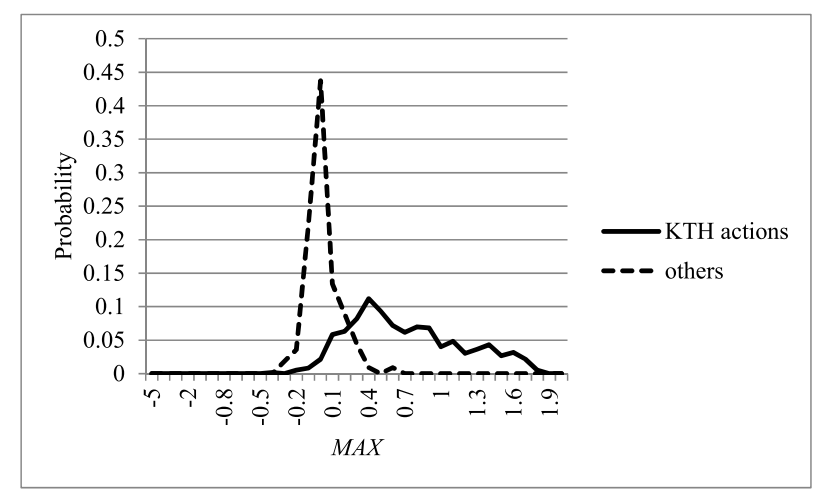

Fig. 9 Distribution of maximum score values $M A X$ for videos of the six $\mathrm{KTH}$ actions and the other types of actions, respectively. sion matrix of NBMIM_f $+\varepsilon$-NN+Var for UCF sport dataset.

\subsection{Action Recognition with Rejection}

Here, a dataset containing videos of other types of actions is discussed, that is, different classes of actions compared to the six actions of the KTH dataset. To cope with this condition, Eq. (8) with rejection is used. One hundred and twenty videos of other types of actions were collected from Weizmann, UCF sport action, UCF YouTube action datasets or through Google video retrieval for experiments. Figure 8 shows some examples of these videos. Figure 9 presents the probability distribution of maximum score values $M A X$ in Eq. (8) for videos of the six KTH actions and the other types of actions, respectively. Figure 9 shows that the proposed action recognition has the discriminative power to verify whether or not the actions belong to the classified actions since the distribution is bimodal. Figure 10 presents ROC curves achieved by NBMIM_f $+\varepsilon-\mathrm{NN}+\operatorname{Var}$ with $S=24$ for various threshold values of $\mathrm{Score}_{h}$ on the KTH dataset and the dataset including other types of actions, respectively. In addition, Fig. 11 presents comparison of recall, precision, and F-score with $S=24$ for various threshold values of

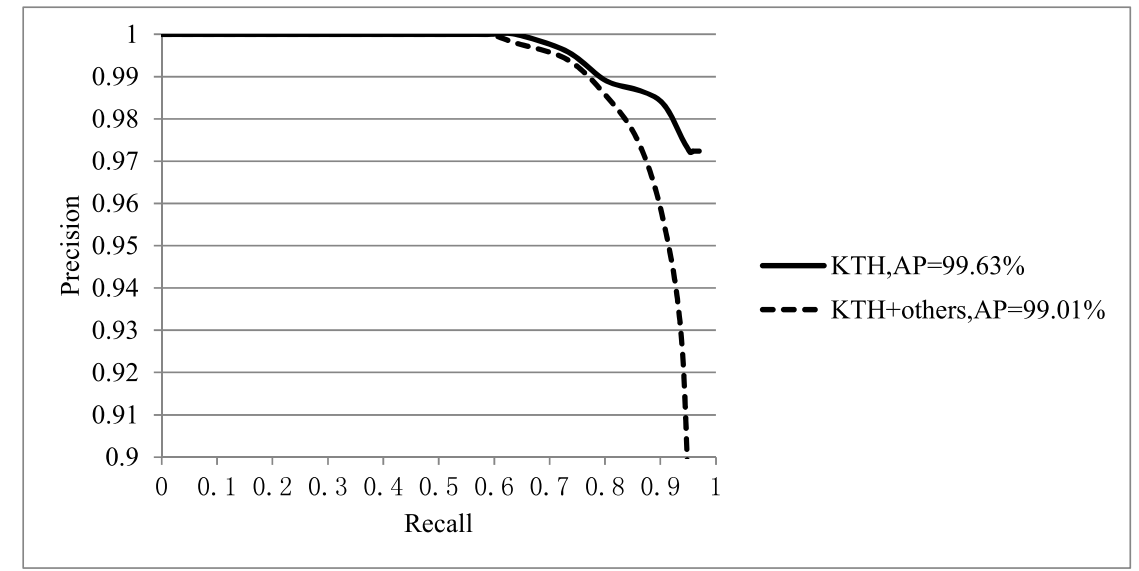

Fig. 10 ROC curves achieved by NBMIM_f $+\varepsilon-\mathrm{NN}+$ Var with $S=24$ for various threshold values of $\mathrm{Score}_{h}$ on the KTH dataset and the dataset including other types of actions, respectively.

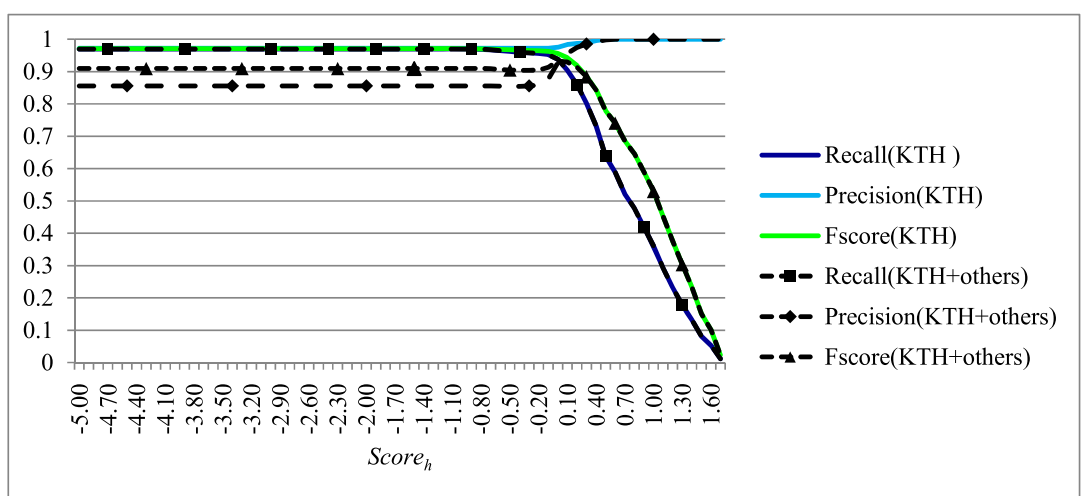

Fig. 11 Comparison of recall, precision, and F-score with $S=24$ for various threshold values of $\mathrm{Score}_{h}$ on the KTH dataset and the dataset including other types of actions, respectively. 
Score $_{h}$ on the KTH dataset and the dataset including other types of actions, respectively. Figures 9 and 11 indicate that the optimal threshold value of $\mathrm{Score}_{h}$ is close to 0 .

\section{Conclusions}

This work proposes the $\varepsilon$-NN probability estimation method and variance filter for STIP selection to improve the accuracy of action recognition. Experimental results reveal that the proposed method has classification accuracies of 95.37 and $97.00 \%$ for the KTH dataset with 16 and 24 training subjects, respectively. These accuracies exceed the 93.52 and $94.00 \%$ achieved using the original NBMIM (NBMIM_a+1-NN) [23]. Additionally, to the best of the authors' knowledge, the best accuracies that have been achieved using the-state-of-art methods for the KTH dataset with 24 training subjects are $95.33 \%$ [27], 95.10\% [28], and $94.20 \%$ [29]. Thus, the method proposed in this work is superior to the existing action recognition methods.

The method proposed in this work has some limitations, for example our method works only in the case that one person performs one action in videos. Therefore, future works should address the following topics. The proposed method should be extended to detect multiple action events in videos such as: more than one action occurring at the same time, more than one action occurring in sequence, or action interactions occurring between humans, or humans and objects.

\section{Acknowledgments}

The authors would like to thank the anonymous reviewers for the valuable and insightful comments on the earlier version of this manuscript. This work was partially supported by National Nature Science Foundation of China (No. 61202143), Doctoral Program Foundation of Institutions of Higher Education of China (No. 20090121110032), Shenzhen Science and Technology Research Foundation (No. JC200903180630A, ZYB200907110169A), and National Science Council of Taiwan (NSC-101-2221-E-155060).

\section{References}

[1] J.K. Aggarwal and M.S. Ryoo, "Human activity analysis: a review," ACM Comput. Surv., vol.43, no.3, pp.1601-1647, 2011.

[2] R. Roppe, "A survey on vision-based human action recognition," Image and Computing, vol.28, no.3, pp.976-990, 2010.

[3] A. Bobick and J. Davis, "The recognition of human movement using temporal templates," IEEE Trans. Pattern Anal. Mach. Intell., vol.23, no.3, pp.257-267, 2001.

[4] Y. Wang and G. Mori, "Human action recognition by semi-latent topic models," IEEE Trans. Pattern Anal. Mach. Intell., vol.31, no.10, pp.1762-1774, 2009.

[5] D. Weinland and E. Boyer, "Action recognition using exemplarbased embedding," Proc. Intl. Conf. Computer Vision and Pattern Recognition, 2008.

[6] L. Wang and D. Suter, "Learning and matching of dynamic shape manifolds for human action recognition," IEEE Trans. Image Process., vol.16, no.6, pp.1646-1661, 2007.
[7] J.-W. Hsieh, Y.-T. Hsu, H.-Y. Mark, Liao, and C.-C. Chen, "Videobased human movement analysis and its application to surveillance systems," IEEE Trans. Multimedia, vol.10, no.3, pp.372-384, 2008.

[8] I. Laptev, "On space-time interest points," Intl. J. Comput. Vis., vol.64, no.2-3, pp.107-123, 2005.

[9] C. Harris and M. Stephens, "A combined corner and edge detector," Proc. Alvey Vision Conference, 1988.

[10] P. Dollar, V. Rabaud, G. Cottrell, and S. Belongie, "Behavior recognition via sparse spatio-temporal features," Proc. Intl. Conf. Visual Surveillance and Performance Evaluation of Tracking and Surveillance, 2005.

[11] G. Willems, T. Tuytelaars, and L. Van Gool, "An efficient dense and scale-invariant spatio-temporal interest point detector," Proc. European Conf. Computer Vision, 2008.

[12] P. Scovanner, S. Ali, and M. Shah, "A 3-dimensional sift descriptor and its application to action recognition," Proc. Intl. Conf. Multimedia, 2007.

[13] J.C. Niebles, H. Wang, and F.-F. Li, "Unsupervised learning of human action categories using spatial-temporal words," Int. J. Comput. Vis., vol.79, no.3, pp.299-318, 2008.

[14] J. Liu and M. Shah, "Learning human actions via information maximization," Proc. Intl. Conf. Computer Vision and Pattern Recognition, 2008

[15] H. Wang, M. Ullah, A. Klaser, I. Laptev, and C. Schmid, "Evaluation of local spatio-temporal features for action recognition," Proc. British Machine Vision Conference, 2009.

[16] I. Laptev and T. Lindeberg, "Local descriptors for spatio-temporal recognition,” Proc. Intl. Workshop Spatial Coherence for Visual Motion Analysis, 2004.

[17] I. Laptev, M. Marszałek, C. Schmid, and B. Rozenfeld, "Learning realistic human actions from movies," Proc. Intl. Conf. Computer Vision and Pattern Recognition, 2008.

[18] A. Kläser, M. Marszałek, and C. Schmid, "A spatio-temporal descriptor based on 3D-gradients," Proc. British Machine Vision Conference, 2008.

[19] T. Ogata, W. Christmas, J. Kittler, and S. Ishikawa, "Improving human activity detection by combining multi-dimensional motion descriptors with boosting," Proc. Intl. Conf. Pattern Recognition, 2006.

[20] I. Laptev and P. Pérez, "Retrieving actions in movies," Proc. Intl. Conf. Computer Vision, 2007.

[21] Y. Wang and G. Mori, "Learning a discriminative hidden part model for human action recognition," Proc. Neural Information Processing Systems, pp.1721-1728, 2008.

[22] O. Boiman, E. Shechtman, and M. Irani, "In defense of nearestneighbor based image classification," Proc. Intl. Conf. Computer Vision and Pattern Recognition, 2008.

[23] J. Yuan, S. Liu, and Y. Wu, "Discriminative video pattern search for efficient action detection," IEEE Trans. Pattern Anal. Mach. Intell., vol.33, no.9, pp.1728-1743, 2011.

[24] H. Jhuang, T. Serre, L. Wolf, and T. Poggio, "A biologically inspired system for action recognition," Proc. Intl. Conf. Computer Vision, 2007.

[25] S. Nowozin, G. Bakir, and K. Tsuda, "Discriminative subsequence mining for action classification," Proc. Intl. Conf. Computer Vision, 2007.

[26] http://www.nada.kth.se/cvap/actions/

[27] T. Zhang, J. Liu, S. Liu, C. Xu, and H. Lu, "Boosted exemplar learning for action recognition and annotation," IEEE Trans. Circuits Syst. Video Technol., vol.21, no.7, pp.853-866, 2011.

[28] H.J. Seo and P. Milanfar, "Action recognition from one example," IEEE Trans. Pattern Anal. Mach. Intell., vol.33, no.5, pp.867-882, 2011.

[29] H. Wang, A. Klaser, C. Schmid, and C.-L. Liu, "Action recognition by dense trajectories," Proc. Intl. Conf. Computer Vision and Pattern Recognition, 2011.

[30] M.D. Rodriguez, J. Ahmd, and M. Shah, "Action match: A spatio- 
temporal maximum average correlation height filter for action recognition," Proc. Intl. Conf. Computer Vision and Pattern Recognition, 2008.

[31] A. Kovashka and K. Grauman, "Learning a hierarchy of discriminative space-time neighborhood features for human action recognition," Proc. Intl. Conf. Computer Vision and Pattern Recognition, 2010.

[32] Q. Le, W. Zou, S. Yeung, and A. Ng, "Learning hierarchical invariant spatio-temporal features for action recognition with independent subspace analysis," Proc. Intl. Conf. Computer Vision and Pattern Recognition, 2011.

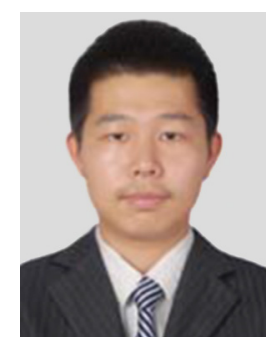

Hongbo Zhang received the B.S. degree in Computer Science and Technology from Shenyang Normal University, Shenyang, China, in 2008. He is currently working toward Ph.D. degree in artificial intelligent in School of Information Science and Technology of Xiamen University, China. During Sept. 2011 and July 2012, he went to Yuan Ze University as an exchange student. His research interests include human action analysis, object detection/recognition, and image/video retrieval.

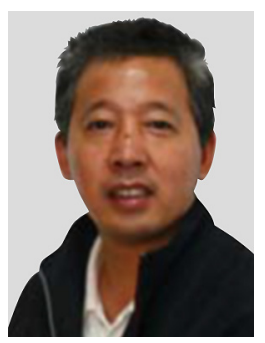

Shaozi Li received the B.S. degree from the Computer Science Department, Hunan University in 1983, and the M.S. degree from the Institute of System Engineering, Xi' an Jiaotong University in 1988, and the Ph.D. degree from the College of Computer Science, National University of Defense Technology in 2009. He currently serves as the Professor and Chair of School of Information Science and Technology of Xiamen University, the Vice Director of Fujian Key Lab of the Brain-like Intelligence System, and the Vice Director and General Secretary concurrently of the Council of Fujian Artificial Intelligence Society. His research interests cover artificial intelligence and its applications, moving objects detection and recognition, machine learning, computer vision, natural language processing and multimedia information retrieval, network multimedia and CSCW technology and others.

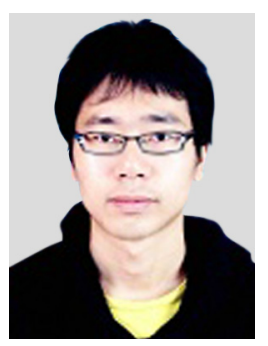

Songzhi Su received the B.S. degree in Computer Science and Technology from Shandong University, China, in 2005. He received M.S. and Ph.D degree in Computer Science in 2008 and 2011, both from Xiamen University, Fujian, China. He joined School of Information Science and Technology of Xiamen University as an assistant professor in 2011. His research interests include pedestrian detection, time-of-flight camera based human action recognition and image/video retrieval.

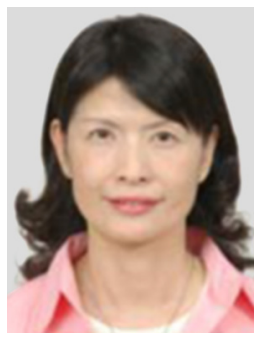

Shu-Yuan Chen received the B. S. degree in Electrophysics in 1980, the M. S. and Ph.D. degrees both in Computer Engineering in 1982 and 1990, all from National Chiao Tung University, Hsinchu, Taiwan. Dr. Chen joined the faculty of Yuan Ze University (YZU), Taoyuan, Taiwan, in 1994 and has been an YZU Professor in the Department of Computer Science and Engineering from 2002. At YZU, Professor Chen has been the Head of the Department of Computer Science and Engineering from 2002 through 2004.

Professor Chen's major research interests include image processing, pattern recognition, intelligent transportation systems, and image/video retrieval. 